\title{
Characteristics of cases of fatal drowning at a tertiary care hospital in Sri Lanka
}

\author{
Ariyarathna HTDW, Jayathilaka ELPA \\ Department of Forensic Medicine, Faculty of Medical Sciences, University of Sri Jayewardenepura, Gangodawila, \\ Nugegoda, Sri Lanka.
}

Correspondence: $\quad$ Dr. H. T. D. W. Ariyarathna

e-mail: ariyaratna@sjp.ac.lk

https://orcid.org/0000-0002-4266-5508

Submitted on 20.01.2021 and accepted for publication on 11.05.2021

\begin{abstract}
Introduction: Deaths due to drowning largely manifest as preventable accidents or suicides. This study deals with characteristics of cases of fatal drowning presented to a tertiary care hospital in Sri Lanka.
\end{abstract}

Methods: A descriptive study was conducted retrospectively based on postmortem reports of deaths due to drowning available at the Colombo South Teaching Hospital from September 2015 to September 2020. The study reviews characteristics of fatal drowning cases reported to the institution including their epidemiology, features, injury patterns and associated factors.

Results: Drowning accounted for $1.35 \%(n=65)$ of total postmortems performed. The male to female ratio was $8: 1$. Children below 15 years of age contributed to $7.7 \%$ of the deaths. Accidental deaths were the commonest followed by suicide and homicide respectively. Evidence of alcohol consumption was noted in $30.8 \%(\mathrm{n}=20)$ and the majority of the drownings occurred in the sea. No putrefaction was seen in $58.5 \%$ $(\mathrm{n}=38)$ bodies. The characteristic froth was noted in $43.1 \%(\mathrm{n}=28)$ of the study sample and there had not been a significant association between the presence of froth with the medium of water. Facial and chest injuries were the commonest associated injuries. Four cases of late death (near drowning) were reported. Twenty-six (40\%) deaths could have been prevented.

Conclusions: This study helps to emphasize an existing problem in Sri Lanka. The prevention of drowning is mostly an achievable goal in Sri Lanka as long as the risk factors and epidemiology are known along with an adequate emphasis.

Key words: Drowning, epidemiology, prevention

\section{Introduction}

Deaths due to drowning is not unusual to a forensic pathologist. Deaths that ensue submergence of a person in water is considered as drowning. When the manner or circumstance of death is considered, drowning could be accidental, suicidal, homicidal, or unascertained. Drowning leads to different outcomes. Some victims who drown mange to survive without any ill effects. The events of drowning may cause immediate death, delayed death, morbidity or even brain death resulting in a vegetative status. The latest Sri Lankan Annual Health Bulletin (2019) does not specifically mention the mortality and morbidity data of immersion incidents. (It mentions only overall 'injury' data that include poisoning and other consequences of external causes (S00-T98) (1). 
The Indoor Morbidity and Mortality Record (IMMR) is one of the most important records available in the country for the evaluation of the health status of Sri Lanka. Indoor Morbidity and Mortality Reporting of Sri Lanka should be commended at this point, as it attempts to classify injuries and diseases according to the International Statistical Classification of Diseases and Related Health Problems (ICD), but with certain deficiencies. Drowning incidents are just grouped under injuries in the IMMR, but no real picture or the ICD are depicted therein (2). Moreover, an agreed terminology in relation to drowning is essential for an effective comparison to be done (3). According to the World Health Organization (WHO), though drowning is easily preventable, an adequate study and consideration have not been given to this subject. Even global estimations are considered to be significantly lower than the real incidence of drowning (4).

The author is of the view that there should be a readily available data set for fatal drowning in a country, as some of the cases are easily preventable. Furthermore, the existing patterns should be available before implementing any of the preventive measures. Following postmortem examinations, ways of preventability from drowning were considered. This exercise showed that most of the deaths were unwarranted. Although the underlying causes for drowning are multifactorial, it is always beneficial to have a detailed national database, as many strategies can be implemented to reduce the incidence of such tragedies. However, there is no national database on deaths due to drowning in Sri Lanka. Furthermore, there is no proper emphasis on preventability and no detailed reports or published studies are available highlighting the aspect of preventability.

This study was primarily designed to study characteristics of cases of fatal drowning reported to the institution, encompassing their epidemiology, characteristic features, injury patterns and associated factors.

\section{Methods}

A descriptive study was carried out retrospectively based on the reports of postmortem examinations performed at the Colombo South Teaching Hospital from September 2015 to September 2020. Postmortem records of all confirmed cases of deaths due to drowning during the study period were selected for the study. The study reviewed characteristics of fatal drowning including its epidemiology, features, injury patterns and associated factors. Data was analysed with SPSS $^{\mathrm{TM}}$ version 21 and $p<0.05$ was considered as statistically significant. Ethical approval for the study was obtained from the Ethics Review Committee of Colombo South Teaching Hospital.

\section{Results}

A total number of 4804 postmortem examinations were performed during a period of 5 years. Out of them, 65 deaths were due to drowning and they accounted for a $1.35 \%$ of all the postmortem examinations performed. There were 58 males and 7 females within the study sample (male : female ratio $8: 1)$. There were only four incidents, where the victims died only following admission (near drowning). All the other victims were dead on admission $(\mathrm{n}=61,93.8 \%)$. The mean age was 42.7 $(\mathrm{SD} \pm 23.53)$ years. The highest number of deaths were reported in the age category of above 70 years followed by the age category of $16-20$ years showing a biphasic distribution. For the ease of comparison with other studies done locally and internationally, the age groups were divided into three classes; less than 15 years, 16 - 30 years and more than 30 years. The highest number of deaths $(\mathrm{n}=38,58.5 \%)$ were reported in the above 30-year age group (Table 1).

Table 1: Age distribution of the study sample

\begin{tabular}{lcc}
\hline Age group & $\begin{array}{c}\text { Frequency } \\
\text { (n) }\end{array}$ & $\begin{array}{c}\text { Percentage } \\
(\%)\end{array}$ \\
\hline Below 15 years & 5 & 7.7 \\
16 years - 30 years & 22 & 33.8 \\
Above 30 years & 38 & 58.5 \\
Total & 65 & 100 \\
\hline
\end{tabular}


Table 2: The circumstances/manner of death in the study sample

\begin{tabular}{lcc}
\hline $\begin{array}{l}\text { Circumstances of } \\
\text { death }\end{array}$ & $\begin{array}{c}\text { Frequency } \\
\text { (n) }\end{array}$ & $\begin{array}{c}\text { Percentage } \\
\text { (\%) }\end{array}$ \\
\hline Accidental & 41 & 63.1 \\
Suicidal & 14 & 21.5 \\
Homicidal & 1 & 1.5 \\
Unascertained & 9 & 13.8 \\
Total & 65 & 100.0 \\
\hline
\end{tabular}

Most deaths were accidental $(\mathrm{n}=41,63.1 \%)$, followed by suicides $(\mathrm{n}=14,21.5 \%)$. One death was a homicide $(1.5 \%)$, involving a 43 -year-old male. Nine deaths $(13.8 \%)$ could not be definitively categorized and they were categorized as "unascertained" (Table 2).

There was a considerable gap between males and females in accidental deaths (male to female ratio 7.3:1) and suicidal deaths (male to female ratio 6.1: (1). No females were found in the categories of homicide and unascertained drowning deaths (Figure 1).

\section{State of Alcohol consumption before the drowning}

It was ascertained at the postmortem examination by the time of death that $20(30.8 \%)$ victims had consumed alcohol whereas $30(46.2 \%)$ had not. In 14 cases $(21.5 \%)$ alcohol consumption status before drowning could not be ascertained.

Table 3 outlines the site of drowning and the presence of typical froth. Sea was the commonest location for drowning $(\mathrm{n}=30,46.2 \%)$ followed by wells $(n=16,24.6 \%)$. Other locations included swimming pools, lakes, ponds, and water ditches $(\mathrm{n}=19,29.2 \%)$. Twenty-eight bodies $(43.1 \%)$ showed the presence of typical froth while thirtyfive of them (53.8\%) did not show such froth. There was no significant association between the medium of drowning and the presence of froth around the nose and mouth.

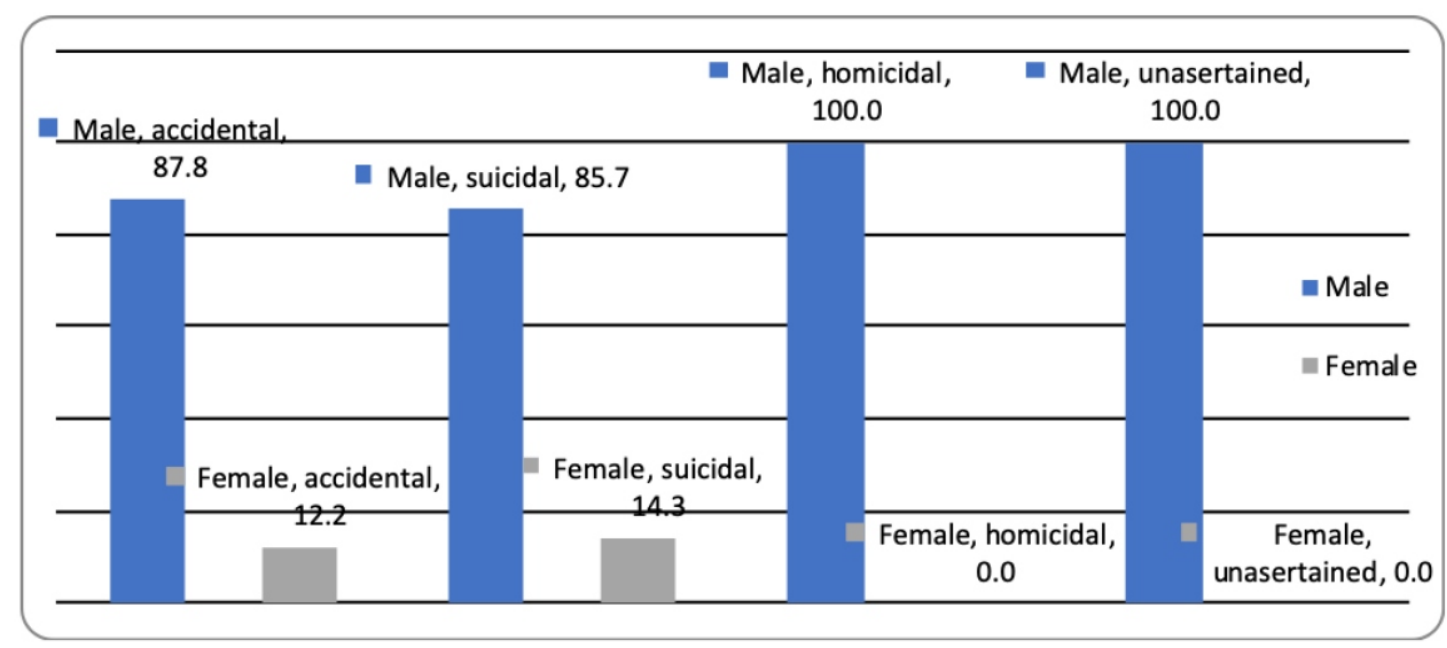

Figure 1: Gender distribution of circumstances/manner of death

Table 3: The association of the site of drowning and the presence of typical froth

\begin{tabular}{lrrrrr}
\hline \multirow{2}{*}{ Factor } & \multicolumn{4}{c}{ Typical froth } & \multirow{2}{*}{ Significance } \\
\cline { 2 - 5 } & \multicolumn{2}{c}{ Yes } & \multicolumn{2}{c}{ No } & \\
\hline Location of drowning & & & & & \\
Sea & 14 & $(50.0 \%)$ & 15 & $(42.9 \%)$ & $p=0.785$ \\
Well & 6 & $(21.4 \%)$ & 10 & $(28.6 \%)$ & \\
Other & 8 & $(28.6 \%)$ & 10 & $(28.6 \%)$ & \\
\hline
\end{tabular}


Table 4: Distribution of antemortem and postmortem injuries on the body

\begin{tabular}{lcccc}
\hline Places & \multicolumn{2}{c}{$\begin{array}{c}\text { Ante-mortem injuries } \\
\text { \% }\end{array}$} & $\begin{array}{c}\text { Postmortem } \\
\text { n }\end{array}$ & $\begin{array}{c}\text { injuries } \\
\text { \% }\end{array}$ \\
\hline Face & 9 & 56.25 & 5 & 27.77 \\
Limbs & 5 & 31.25 & 8 & 44.44 \\
Abdomen & - & - & 2 & 11.11 \\
Chest & 2 & 12.50 & 3 & 16.66 \\
Total & 16 & 100 & 18 & 100 \\
\hline
\end{tabular}

* Some bodies had both antemortem and postmortem injuries, where as 41 bodies did not have any injury

Antemortem and/ or postmortem injuries were noted in $24(36.9 \%)$ individuals at sites such as the face, chest, abdomen, and limbs. No injuries were noticed in 41 (63.1\%) bodies. When considering antemortem injuries alone, face was the commonest region affected $(n=9,56.25 \%)$ followed by the limbs $(\mathrm{n}=5,31.25 \%)$, whereas in postmortem injuries the limbs $(n=8,44.44 \%)$ were the commonest body region affected followed by the face $(n=5,27.77 \%)$. Injuries seen over the abdomen were solely of postmortem in origin and this was the body region that had minimally been affected when considering all injuries. When considering each body region and comparison between antemortem and postmortem injuries, the face received more antemortem injuries compared to postmortem injuries (9 vs, 5), and the limbs received more postmortem injuries compared to antemortem injuries (8vs. 5).

There had been four live admissions (6.2\%) following the incident of immersion (near drowning) and later they had succumbed to injuries.

At the time of the postmortem examination, mild/moderate putrefaction and severe putrefaction were noticed in $29.2 \%(n=19)$ and $12.3 \%(n=8)$ of the cases respectively.

Of the 65 cases studied $40 \%(n=26)$ were identified by the respective forensic pathologists as preventable deaths while $12.3 \%$ deaths were identified as unpreventable.

\section{Discussion}

According to the WHO, drowning is considered as the third leading cause of death due to unintentional injury worldwide and it is responsible for $7 \%$ of all injury-related deaths (5). Drowning in general is an area to which a lesser degree of emphasis has been paid to compile accurate data $(4,5)$. Some key characteristics of fatal drowning were identified in this study. Drowning-related incidents are handled under the category of preventable injuries in Sri Lanka, and it is also included as a type of unintentional injury along with road traffic accidents, poisoning, falls, and burns (6).

Drowning is a major public health problem and thus an effective preventive mechanism is mandatory (7). To achieve this, it should include the study all cases of drowning in a given country detailing underlying causes and/or risk factors. That task is impossible when there are no accepted definitions with consistent coding that can be compared locally and internationally. Therefore, a proper data analysis system is needed to be implemented in our country. From a definitional perspective, the WHO define drowning as "death by asphyxia due to submersion in a liquid medium." Near-drowning is defined as "immediate survival after asphyxia due to submersion." (8) It may end up with either survival or death (9).

Since there are no accurate statistics in Sri Lanka on drowning-related deaths, it is worthwhile discussing about the victims of drowning as a whole. Some victims do not warrant any hospital admission though they actually experienced the eminent threat. 
The rest of the patients may need hospital admissions. Out of those, a proportion of patients may not experience any ill effect and they recover without any residual damage ("live discharges"). Another proportion of patients may experience permanent brain damage resulting in delayed death. In a real sense, those findings are not counted as cases of drowning. This partially explains why the real number of victims who experienced drowning may be far more than the figures reported in hospital statistics. The author is of the view that not only the fatal cases but also these types of cases too need to be analysed to study its epidemiology and the underlying factors for drowning. It is the responsibility of the forensic pathologists to highlight such important aspects to the stakeholders. The IMMR is prepared by using statistics provided by government hospitals. But according to the Annual Health Statistics of Sri Lanka, around 60\% of deaths are encountered in non-hospital settings. Such facts should also be kept in mind while considering the national statistics (1).

The present study revealed that fatal drowning constitutes a little more than one percent of the total postmortem examinations conducted at the institution during the given period. There were two peaks observed in the age distribution. According to the author's experience, the first peak in the 16-20 years age group could have been due to the reason that young males tend to consume alcohol and swim during their leisure time causing drowning. The second peak may be due to suicides and accidental causes with advancing age.

The WHO state that nearly $60 \%$ of all drowning deaths were "below the age of 30 years" (4). In our sample, only a $41.54 \%(n=27)$ were below 30 years. The number of children below 15 years was 5 (7.7\%). A study done in Australia has revealed that the maximum morbidity of children of $0-4$ years is 2.63 per 100,000 peoplen (10). The findings of our study is also compatible with a previous study done in Sri Lanka and indicated the lowest drowning rate in the 5-14 year age group (11).

A male preponderance was observed in this study under discussion and the same is confirmed with both local and international literature. In a crosscountry analysis performed in Sri Lanka by Mathews et al, it was revealed as 4:1 (12). According to a systematic review, males are at greater risk, and it accounted for 45,240 events (75\%) compared to 15,295 female events $(25 \%)$ from 50 out of 62 studies (13).

The manner/circumstances of death play a significant role in deaths due to drowning. All manners of death presented here are commoner in males with a considerable gap between females. A systematic review by Tyler et al., who studied the epidemiology of drowning in low- and middleincome countries also showed that accidental death is the most common category in all instances studied (14). A study done in in Sri Lanka has revealed that the commonest circumstance was accidental as expected and it amounted to $57.6 \%$, while suicide $22 \%$. In the same study, circumstances were unascertained in $20.3 \%$ and no homicides were reported(15).

Colombo is a city where the sea is closer to any given point and it could well be the most common site for drowning. Out of the accidental deaths, two deaths were due to drowning in swimming pools; a five-year-old boy while participating in a wedding ceremony with his parents, had accidentally drowned. The second swimming pool death was an accidental drowning after alcohol consumption and the victim was also a male of thirty years. The author wishes to highlight the preventability of these two deaths. With a four-sided fence and parental supervision, the death of this 5-year-old child could have been prevented. Related laws of swimming pool safety have not been implemented in Sri Lanka yet. The United States Centers for Disease Control and Prevention (CDC) has identified various predisposing factors related to swimming pool deaths and injuries. Subpar swimming skills, absence of four-sided fencing around the pool, lack of supervision, alcohol use and seizure disorders are some of the risk factors (16). Stakeholders should implement strict laws at swimming pool facilities in Sri Lanka along with four-sided fencing. It is recommended to employ a lifeguard/ pool attendant as well.

The study identified four cases with some survival time following the initial incident of drowning. Such cases are termed as cases of "near drowning". The majority had reportedly died during the incident. One case had been treated for a day in a ward and 
the other three had only been treated for a few hours. The management of cases of near drowning may need ventilation and it is still useful to have statistics in those victims as well (17).

The cause of death of drowning is ascertained by excluding all other possible causes. The presence of typical froth is considered more or less a specific feature in drowning. The present study revealed the typical froth in a little less than half of the deaths. Fine, white, coarse and copious froth is considered as a significant finding in relation to deaths due to drowning. It was attempted to find whether there is a significant difference in presence of froth depending on the medium of drowning. No significant difference was identified. In a study done in the Netherlands, only $16.1 \%$ of cases had shown the characteristic froth and it was only in freshwater drowning (18).

Alcohol consumption before death was identified in approximately one third of individuals and thirty individuals had not consumed any alcohol in our study. Since there was no reliable evidence, it was not possible to comment on the remainder of the study sample. According to the Ministry of Health Sri Lanka, human behaviour is identified as a key risk factor for the occurrence of drowning (6). In another Sri Lankan study, $21 \%$ of individuals had consumed alcohol before accidental drowning (15).

Decomposition is a frequent occurrence observed in dead bodies that are recovered from water. Only a $41.5 \%$ of dead bodies showed putrefactive changes in our study. Decomposition changes were noticed in $52 \%$ of cases in a 10-year retrospective study done in India (19).

A dead body recovered from water may show injuries either in the forms of antemortem or as postmortem injuries. According to Farrugia et al., traumatic injuries are not common in subjects with a history of drowning. Only seven of them had sustained traumatic soft tissue injures (20). Fornes et al., have emphasized that differentiation between antemortem and postmortem injuries is difficult at times due to multitude of reasons. The absence of well-defined criteria is one such reason (21). The analysis of postmortem injures sustained with immersion is also helpful for forensic pathologists during the exercise of injury interpretation. Apart from macroscopy, histology also plays an important role in differentiation of antemortem and postmortem injuries. In non-putrefied dead bodies microscopic examination of body parts may contribute to differentiate antemortem drowning from postmortem drowning. Histological examination of lung tissues may provide clues. In addition to trauma, state of the dress, effort made to rescue the body, adiposity, postmortem contact of the body against surfaces, animal predation and the amount of air in the lungs, and bowels before drowning are a few factors that determine the nature of postmortem injury patterns (22).

In Australia, with more or less the same population as in Sri Lanka, numbers of drowning deaths were very low, despite the population engaging more in water sports and water-related activities (23). The Sri Lanka Life Saving, Life Saving Victoria in Australia and the Australian High Commission in Sri Lanka have declared 3.5 deaths per 100,000 people in Sri Lanka from 2016-2018 (24). However, the present study deals with small numbers and therefore comparison is not feasible.

One of the key concerns of this study is to study the preventability of drowning in the cases studied. The respective forensic pathologists have mentioned that $40 \%$ of the cases could have been prevented and $12.3 \%$ deaths have been identified as unpreventable. The death of young individuals who can contribute to the economy of the country negatively affects the work force. According to the latest reports available in Sri Lanka, about 800 Sri Lankans annually die due to drowning and it is the second leading cause for deaths due to unintentional injury (6). Therefore, forensic pathologists must perform evidence-based studies to convey the important aspects of drowning to prevent such unwarranted deaths.

\section{Recommendations}

- The relevant age groups are to be addressed to prevent premature/ unnecessary deaths.

- The vulnerable areas of the sea should be secured with much wider publicity.

- Strict rules and regulations should be imposed to prevent swimming pool-related deaths. 
- The underlying causes/ risk factors contributing to the death of children by drowning should be studied.

- The contribution of alcohol consumption to drowning needs to be studied quantitatively or by performing blood tests in every possible case.

- The exact burden of deaths due to drowning is to be identified with more emphasis to develop accurate databases representing the whole country.

- A compulsory and detailed reporting system of all the deaths due to drowning with possible risk factors should be implemented and a national database needs to be maintained.

- Cases of near-drowning and live discharges following immersion incidents too need to be included for the drowning statistics of the country with uniform definitions, to be on par with the international nomenclature.

\section{Conclusions}

One of the most important facts that were highlighted in this study is that a little less than the half of deaths studied could have been prevented. The data collection and analysis related to drowning is not yet carried out, up to a desired standard in order to arrest and prevent the existing threats effectively in Sri Lanka. Under injury prevention strategies, drowning is a preventable type of death. Therefore, a proper emphasis must be paid for taking preventive measures through a careful analysis of details surrounding the case. A Judicial Medical Officer/ Forensic pathologist certainly can contribute to achieving this target as long as a proper emphasis is given.

\section{Limitations}

The sample size of the study is a major limitation. The study reflects only one tertiary care hospital in the Western province of the country making it impossible to generalize the study findings.

\section{Acknowledgments}

Dr. Samitha Sirithunga, (Consultant Community Physician, National Programme Manager (Injury
Prevention), Ministry of Health, Mr. Sithum Manjika (Statistical officer, Department of Community Medicine, University of Sri Jayewardenepura), Dr. P. Dasanayaka and Dr. S.R. Hulathduwa (Consultant Forensic Pathologists), Dr.P.H. Jayawardana, Dr. R.M.T.S. Weerasingha, Dr. P.A.C.T. Kumarasiri, Dr. T.N.R.D. Mal, Dr. S.P.R. Siriwardene, Dr. K.L.S.V. Karandana former demonstrators attached to the Department of Forensic Medicine, University of Sri Jayewardenepura, Sri Lanka.

\section{References}

1. Ministry of Health [Internet]. [cited 2021 Aug 16]. Available from: http://www.health.gov.lk/moh_final /english/others.php?pid $=110$

2. AHS 2019.pdf [Internet]. [cited 2021 Aug 16]. Available from: http://www.health.gov.lk/moh_final/ english/public/elfinder/files/publications/AHB/AHS\%20 2019.pdf

3. Final_report_full_web.pdf [Internet]. [cited 2021 Aug 23]. Available from: https://www.who.int/violence_injury_ prevention/global_report_drowning/Final_report_full_we b.pdf

4. Drowning. [cited 2020 Sep 15]. Available from: https://www.who.int/news-room/fact-sheets/detail/ drowning

5. Drowning [Internet]. [cited 2021 Aug 16]. Available from: https:/www.who.int/news-room/fact-sheets/detail/ drowning

6. Sri Lanka : About 800 people drown annually in Sri Lanka Health Ministry [Internet]. [cited 2021 Aug 16]. Available from: http://www.colombopage.com/archive_21A/ Jul24_1627137929CH.php

7. Swimming Injury Statistics - Swimming Pool Accidents [Internet]. edgarsnyder.com. [cited 2021 Aug 16]. Available from: https://www.edgarsnyder.com/statistics/ swimming-pool-statistics.html

8. Drowning [Internet]. [cited 2020 Sep 19]. Available from: https://www.who.int/westernpacific/health-topics/ drowning

9. Busuttil A, Keeling J. Paediatric Forensic Medicine and Pathology. CRC Press; 2008.503 p.533. 
10. Franklin RC, Scarr JP, Pearn JH. Reducing drowning deaths: the continued challenge of immersion fatalities in Australia. Med J Aust. 2010 Feb 1; 192(3): 123-6.

11. Sri Lanka sinks in water safety; over 1,100 deaths by drowning each year | Daily FT [Internet]. [cited 2020 Sep 16]. Available from: http://www.ft.lk/special-report/SriLanka-sinks-in-water-safety--over-1-100-deaths-bydrowning-each-year/22-645877.

12. Matthews B, Birch R, Jayawardena M, Mathew D, Nanayakkara A, Wiyayaratne $\mathrm{S}$, et al. A cross country analysis of drowning in Sri Lanka: 2001 to 2006 and 2009. Inj Prev. 2016 Sep; 22(Suppl 2): A295.1-A295.

13. Cenderadewi M, Franklin R, Peden A, Devine S. Fatal intentional drowning in Australia: A systematic literature review of rates and risk factors. PLOS ONE. 2020; 15(5): e0231861.

14. Tyler MD, Richards DB, Reske-Nielsen C, Saghafi O, Morse EA, Carey R, et al. The epidemiology of drowning in low- and middle-income countries: a systematic review. BMC Public Health. 2017 May 8; 17(1): 413.

15. Paranitharan P, Perera W, Lakmal S, Priyanath D, Senanayake T, Kumari M. Deaths following drowning in Sri Lanka - a retrospective study. Sri Lanka Journal of Forensic Medicine, Science \& Law. 2020; 11(2): 1. http://doi.org/10.4038/sljfmsl.v11i2.7857.

16. Swimming Injury Statistics - Swimming Pool Accidents [Internet]. edgarsnyder.com. [cited 2021 Aug 16]. Available from: https://www.edgarsnyder.com/statistics/ swimming-pool-statistics.html

17. Drowning (submersion injuries) - UpToDate [Internet] [cited 2021 Aug 16]. Available from: https://www.uptodate.com/contents/drowningsubmersion-injuries
18. Reijnen G, Buster MC, Vos PJE, Reijnders UJL. External foam and the post-mortem period in freshwater drowning; results from a retrospective study in Amsterdam, the Netherlands. J Forensic Leg Med. 2017 Nov 1; 52: 1-4.

19. Mohanty SK, Kumar V, Hussain APJ, Bhuvan V. Epidemiological analysis of drowning deaths: A 10 year Study. J Indian Acad Forensic Med. 2016; 38(4): 465.

20. Farrugia A, Ludes B. Diagnostic of Drowning in Forensic Medicine. ResearchGate. 2010 [cited 16 May 2021]. Available from: https:/www.researchgate.net/publication/ 221916186_Diagnostic_of_Drowning_in_Forensic_Medi cine)

21. Fornes P, Pépin G, Heudes D, Lecomte D. Diagnosis of Drowning by Combined Computer-Assisted Histomorphometry of Lungs with Blood Strontium Determination. Journal of Forensic Sciences. 1998; 43(4): 14305J.

22. Armstrong EJ, Erskine KL. Investigation of Drowning Deaths: A Practical Review. Acad Forensic Pathol. 2018 Mar; 8(1): 8-43.

23. Cenderadewi M, Franklin R, Peden A, Devine S. Fatal intentional drowning in Australia: A systematic literature review of rates and risk factors. PLOS ONE. 2020; 15(5): e0231861

24. Unintentional Drowning: Get the Facts | Home and Recreational Safety | CDC Injury Center. Cdc.gov. [cited 16 May 2021]. Available from: https://www.cdc.gov/ homeandrecreationalsafety/water-safety/waterinjuriesfactsheet.html 